\title{
Was auf dem Spiel steht
}

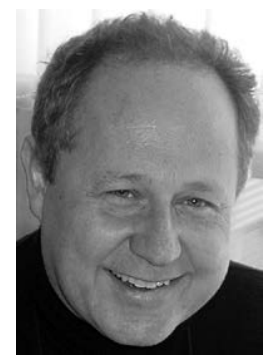

Prof. Dr. med. Manfred Wildner
Korrespondenzadresse

Prof. Dr. med. Manfred Wildner

Bayerisches Landesamt für Lebensmittelsicherheit

Veterinärstraße 2

85764 Oberschleißheim

Manfred.Wildner@Igl.bayern.de

Bibliografie

DOI https://doi.org/10.1055/s-0043-110753

Gesundheitswesen 2017; 79: 451-452

(c) Georg Thieme Verlag KG Stuttgart · New York

ISSN 0941-3790
Die Frage nach dem Sinn bzw. die persönliche Sinnfindung war für den Arzt und Psychiater Viktor Frankl (1905-1997) zentral. Als Überlebender verschiedener Konzentrationslager, in welchen er Eltern, Geschwister und Ehefrau verlor, verarbeitete er seine traumatischen Erfahrungen auf besondere Weise. Für ihn gab es seither nur noch zwei Gruppen von Menschen: Anständige und Unanständige, quer zu allen Grenzziehungen nach Rassen und Nationen. „Seit Auschwitz wissen wir, wessen der Mensch fähig ist. Und seit Hiroshima wissen wir, was auf dem Spiel steht“, wird er zitiert [1]. Für ihn wurde die Frage nach dem Sinn, welcher gefunden werden muss, zur zentralen Frage [2]. Er entwickelte diese Sinnfrage zu einem therapeutischen Konzept, der Sinntherapie (Logotherapie bzw. Existenzanalyse), welche er in seiner psychiatrischen Tätigkeit entfaltete. Er setzte sich dafür ein, die psychiatrisch-psychotherapeutische Arbeit durch eine geistige Dimension zu ergänzen und grenzte sich damit von vielen enger gefassten Sichtweisen in der Psychiatrie ab. Er arbeitete bis zu seinem Tode in Wien, weshalb seine therapeutische Richtung auch als „Dritte Wiener Schule der Psychotherapie“ bezeichnet wird.

In ähnlicher Weise hatte auch Aaron Antonovsky in seiner Studie an überlebenden Frauen der nationalsozialistischen Konzentrationslager den gesundheitsfördernden, salutogenetischen Gehalt der Sinnhaftigkeit, neben den Dimensionen der Vestehbarkeit und Handhabbarkeit, in seinem „Sense of Coherence“ Ansatz beobachtet [3]. Diese salutogenetische Herangehensweise hat in verschiedenen Variationen insbesondere auch für die Gesundheitsförderung und eine Ressourcenorientierung in der Prävention Bedeutung bekommen. Sie wird auch im Tätigkeitsfeld von Public Health als komplementäre Herangehensweise zur Risiko- und Defizitorientierung in Forschung und Praxis geschätzt und über eine individuelle Betrachtungsweise hinaus auf die Gesundheit von Bevölkerungen und Bevölkerungsgruppen ausgeweitet. Bezüge zum Salutogenesekonzept lassen sich implizit auch in den Ansätzen der Ottawa-Charter zu Befähigung (Empowerment) und Teilhabe (Partizipation) finden (siehe auch [4]).

Gleichzeitig ist auch hier ein vernünftiges, dem Menschen und auch sozial gerechtes Maß zu finden. Eine Überforderung individueller Verantwortungsübernahme, vielleicht sogar in Abwälzung von Verantwortung aus dem gemeinschaftlich zu verantwortenden und gestaltenden öffentlichen Raum auf den einzelnen Menschen und seine Privatsphäre, ist abzulehnen [5]. Im Bereich der Gesundheit des Einzelnen wie auch der Gesundheit von Bevölkerungen greifen monokausale Erklärungsmodelle und Kausalzuschreibungen häufig ohnehin zu kurz. Die Weltgesundheitsorganisation zeigt in ihrem Bericht zu sozialen Determinanten gesundheitlicher Ungleichheit diese Zusammenhänge und Wege zu ihrer Überwindung auf [6]. Moderne Ansätze wie Health Impact Assessment erlauben eine systematische Betrachtung der gesundheitlichen Auswirkung z. B. von Projekten im öffentlichen Raum über verschiedenste Vermittlungspfade [7], das diesbezüglich sehr illustrative „Regenbogendiagramm“ von Dahlgren und Whitehead hat seit seiner Erstpublikation weite Verbreitung gefunden [8].

Doch wie verhält es sich mit der Forschung zum Gegenteil von Sinn - zum Unsinn? Hier ist die wissenschaftliche Literatur eher dünn, zumindest mit Bezug zur Gesundheit. Der amerikanische Philosoph Harry Frankfurt beschäftigt sich in seinem viel zitierten Buch auf 80 Seiten mit Unsinn bzw. Humbug (engl. Originaltitel: On Bullshit [9]). Gerne zitiert wird auch Max Blacks Essay zur Prävalenz des Humbugs [10]. Interessant ist dabei die Abgrenzung von Humbug zu einer Lüge: Humbug ist eine Falschdarstellung in trügerischer Absicht, welche jedoch nicht der Sache gilt, sondern vor allem einer, der eigenen Selbstdarstellung dienenden unsinnigen Wiedergabe von Sachverhalten. Prägend hierfür ist nicht die Verneinung der Wahrheit, sondern eine Gleichgültigkeit gegenüber ihr - wer Humbug redet, interessiert sich nicht dafür, ob seine Aussage wahr oder falsch ist. Nach Frankfurts Ansicht ist nicht die Lüge der größte Feind der Wahrheit, sondern letztlich der gegenüber ihr indifferente Humbug (sogenannter „Bullshit“).

Diese Beobachtungen lassen sich in ganz allgemeinen Lebensvollzügen treffen und bestätigen. Von besonderer Relevanz für die Gesundheit werden sie dann, wenn sie im Bereich der Medizin und Gesundheitswissenschaft gemacht werden. Hier sind auch schon erhellende Analysen und Differenzierungen vorgenommen worden, z. B. zur systematischen Verneinung von Evidenz [11], zu pseudowissenschaftlichen Konzeptionen [12], zur industriell orchestrierten Krankheits „erfindung“ mit wirtschaftlichem Eigeninteresse $[13,14]$, zu „Wissenschaftsmüll“ [15] oder auch zu deren gesundheitspolitischen und Public Health-Implikationen [16, 17]. 
Letztlich darf und sollte die Frage nach Sinn und Zweck zu jeder gesundheitsbezogenen Maßnahme nicht nur gestellt werden dürfen, sondern auch gestellt werden. In einer zunehmend mediatisierten Gesellschaft mit wechselseitigen Einflussnahmen von Politik, Massenmedien und Wirtschaft kommt einer ethisch tragfähigen Rechtfertigung der von den verschiedensten Akteuren geführten Reden über Gesundheit und Krankheit große Bedeutung zu [18].

Was dabei auf dem Spiel steht? Der Philosoph Michael Hampe weist scharfsichtig darauf hin, dass sich das auf Wahrheitsfindung ausgerichtete Projekt aufgeklärter Wissenschaft in (mind.) einem Element mit einer sozial verstandenen Demokratie trifft: „Die Überzeugung, dass das Richtige (in einem Fall das gemeinschaftliche Leben, im anderen die richtige Einsicht) nur in einem sehr langen, evtl. Jahrhunderte dauernden kollektiven Streben zu gewinnen ist“ [19]. Und er führt weiter aus, dass eine solche Teilnahme an einem Generationen übergreifenden Projekt, sei es in der Wissenschaft, sei es in einer sozialen Demokratie, in einem sehr positiven Sinn sinnstiftend ist. Seiner Meinung nach richtet ein solcher Handlungsrahmen auf säkulare Weise auf einen überindividuellen Sinn aus und relativiert die Endlichkeit einer partikulären Existenz, vergleichbar einer transzendenten Ewigkeitsperspektive. Die Trivialisierung des Fälschens und Tricksens im Wissenschafts- und Akademiebetrieb durch den Verweis auf das in Wirtschaft und Politik häufig zu Findende markiert für ihn eine besondere Schwere der Verfehlung. Seine Mahnung ist, dass ein „Wissenschaftssystem, das von auf Konkurrenzerfolg geeichten Individuen getragen werden soll, [...] nicht nur seinen Sinn verlieren, sondern vermutlich auch seine finanzielle Basis in der öffentlichen Hand“ verlieren wird [19, S. 491]. Hampe spricht sich für eine Norm- und Ziele-setzende Gemeinschaft aus (Empathie), für die Anerkennung fremder Lebensentwürfe (Toleranz) sowie eine darauf aufbauende Kooperationskompetenz (Vertrauen) als Basis für eine Generationenübergreifende gesellschaftliche Sinngebung.

Dass Große kann sich auch im Kleineren zeigen - in diesem bescheideneren Sinne will auch dieses Heftes wieder zur Erkenntnisgewinnung beitragen: Durch Beiträge über die Delegation ärztlicher Leistungen, die Sichtweise schwangerer Frauen auf ihr Ernährungs- und Bewegungsverhalten, Entscheidungsfaktoren und Informationsquellen für die Krankenhauswahl bei Geburten in der Schweiz, Risikofaktoren der Erwerbsminderungsberentung aufgrund psychischer Erkrankungen, die Gewinnung von JobcenterKlienten für die Teilnahme an einem kardiopräventiven Untersuchungsprogramm, der Erfassung der gesundheitsbezogenen Transitionskompetenz von Jugendlichen mit chronischen Erkrankungen, einer Nutzenbewertung der minimal-invasiven Schulterchirurgie, einer Übersicht zu humanen Expositionen in suizidaler Absicht im Einzugsbereich des Giftnotrufs Erfurt, dem Wissenstransfer eines Konzeptes zur sicheren Patientenidentifikation im Krankenhaus und der Ratenbildung bei KV-Daten mit GKV-Versicherten auf Kreisebene.

Auf diese und andere Weisen lässt sich wissenschaftlich Sinnvolles anbieten - doch kann Sinn in einem tieferen, personalen oder auch gesellschaftlichen Sinn darüber hinaus überhaupt gegeben werden? Viktor Frankl mahnt hier zur Zurückhaltung: „Sinn geben würde auf moralisieren hinauslaufen. Und die Moral im alten Sinn wird bald ausgespielt haben [...] Sinn kann nicht gegeben, sondern muss gefunden werden“ [2]. So bleibt die zentrale Frage, wie solcher - individueller oder auch gemeinschaftlicher - Sinn gefunden werden kann, zunächst offen. Möglicherweise liegt die Antwort in einer Paradoxie: der Sinn besteht vielleicht in dieser individuellen und gesellschaftlichen Suche nach dem Sinn selbst - und darf in Suche und „gefundenem“ Sinn als Pluralität verstanden werden.

Literatur

[1] Fabry JB, FrankI V, Lukas E. Auf den Spuren des Logos. Briefwechsel mit Viktor E. Frankl. Quintessenz Verlag Brlin/München. 1995; S. 147

[2] Frankl V. Der Mensch vor der Frage nach dem Sinn. Eine Auswahl aus dem Gesamtwerk. München: Piper; 2006: S. 155

[3] Antonovksy A. Salutogenese. Zur Entmystifizierung der Gesundheit. Tübingen: DGVT-Verlag; 1997

[4] Franke A. Salutogenetische Perspektive. In: BZgA (Hrsg.) Leitbegriffe der Gesundheitsförderung. URL: http://www.bzga.de/leitbegriffe/?id $=$ angebote\&idx $=164$

[5] Schmidt B. Eigenverantwortung haben immer die Anderen. Der Verantwortungsdiskurs im Gesundheitswesen. Bern: Verlag Hans Huber 2007

[6] CSDH. Closing the gap in a generation: health equity through action on the social determinants of health. Final Report of the Commission on Social Determinants of Health. Geneva: World Health Organization, 2008; URL http://www.who.int/social_determinants/thecommission/ finalreport/en/

[7] Wismar M, Blau J, Ernst K et al., (Hrsg.). Die Wirksamkeit von Gesundheitsfolgenabschätzung. Umfang und Grenzen der Unterstützung von Entscheidungsprozessen in der Europäischen Region. Kopenhagen: WHO, 2007; URL http://www.euro.who.int/document/ E90794.pdf (Zugriff am 15.05.2017)

[8] Dahlgren G, Whitehead M. Policies and strategies to promote social equity in health. Stockholm: Institute for Future Studies, Oxford University Press, 1991; http://heapol.oxfordjournals.org/content/23/5/318.full

[9] Frankfurt H. On Bullshit. Princeton University Press; Princeton New Jersey: 2005

[10] Black M. The prevalence of Humbug. Cornell University Press; Ithaca New York: 1985

[11] Diethelm P, McKee M. Denialism: what it is and how should scientists respond? Eur J Public Health 2009; 19: 2-4

[12] Rupnow D, Lipphardt V, Thiel J et al., (Hrsg.). Pseudowissenschaft. Konzeptionen von Nichtwissenschaftlichkeit in der Wissenschaftsgeschichte. Frankfurt a.M.: Suhrkamp; 2008

[13] Payer L. Disease mongers. New York: John Wiley; 1992

[14] Moynihan R, Heath I, Henry D et al. Selling sickness: the pharmaceutical industry and disease mongering. Commentary: medicalisation of risk factors. Br Med J 2002; 324: 886-891

[15] Macleod MR, Michie S, Roberts I et al. Biomedical research: increasing value, reducing waste. Lancet 2014; 383: 101-104

[16] Kuhn J. Gesundheitspolitik zwischen Evidenzbasierung und Bürgerorientierung. Prävention 2009; 03: 88-92

[17] Paccaud F. Implausible diseases and public health. Eur J Public Health 2007; 17: 410

[18] Schröder-Bäck P, Kuhn J., (Hrsg.). Ethik in den Gesundheitswissenschaften - Eine Einführung. Landsberg: BeltzJuventa; 2016

[19] Hampe M. Warum lügen und betrügen Wissenschaftler? Ein Plädoyer wider die enthemmte Konkurrenz der Einzelkämpfer. Forschung \& Lehre 2016; 23: 490-491 